Seguí-Urbaneja, J.; Inglés, E.; Alcaraz, S. y De Bosscher, V. (2020) Sport Pyramid Metaphor: Trickle Down and Up Effect in Spain. Revista Internacional de Medicina y Ciencias de la Actividad

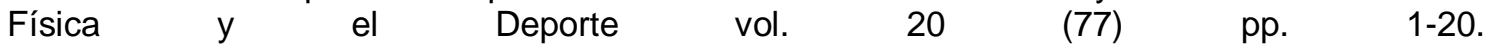
Http://cdeporte.rediris.es/revista/revista77/artmetafora1117.htm DOI: $10.15366 /$ rimcafd2020.77.001

\title{
ORIGINAL
}

\section{LA METÁFORA DE LA PIRÁMIDE DEPORTIVA: EL EFECTO TRICKLE DOWN Y UP EN ESPAÑA}

\section{SPORT PYRAMID METAPHOR: TRICKLE DOWN AND UP EFFECT IN SPAIN}

\author{
Seguí-Urbaneja, J.. ; Inglés, E. ${ }^{2}$; Alcaraz, S. ${ }^{3}$ y De Bosscher, V. ${ }^{4}$ \\ ${ }^{1}$ Dr. Ciencias de la Actividad Física y el Deporte. Instituto Nacional de Educación Física de \\ Cataluña. España. jsegui@inefc.es \\ 2 Dr. Ciencias de la Actividad Física y el Deporte. Instituto Nacional de Educación Física de \\ Cataluña. España. eduard.ingles@gencat.cat \\ 3 Dr. Psicología del Deporte y de la Salud. Universidad Autónoma de Barcelona. España. \\ Saul.Alcaraz@uab.cat \\ 4 Dra. Ciencias Actividad Física y el Deporte. Vrije Universiteit Brussel, Bruselas. Bélgica. \\ Veerle.de.bosscher@vub.ac.be
}

\section{Agradecimientos}

Con el apoyo del Institut Naciona d'Educació Física de Catalunya (INEFC) de la Generalitat de Catalunya.

Código UNESCO: 5902.99 Política Deportiva / Sport policy; 5902 Ciencias políticas / Political sciencies.

Clasificación del Consejo de Europa: 1. Administración, organización y gestión del deporte / Sport administration, organization and management.

Recibido 4 de abril de 2018 Received April 4, 2018

Aceptado 28 de diciembre de 2018 Accepted December 28, 2018

\section{RESUMEN}

La metáfora de la pirámide deportiva (efecto trickle down y trickle up) es utilizada por la administración para justificar la inversión en el deporte de élite. Las evidencias de este efecto son difusas y estudios previos ponen en entredicho su veracidad. Este artículo examina el efecto de esta metáfora en España.

Mediante coeficientes de correlaciones de Spearman ( $\rho)$, se estudia la relación entre el éxito deportivo y la evolución de las licencias federativas de 23 modalidades deportivas, desde 1992 hasta 2016. Los resultados muestran tres grupos distintos: 1) aquellos con indicios de la posible existencia de los efectos 
trickle down y up; 2) aquellos que muestran correlaciones negativas entre número de licencias y éxito deportivo; y 3) aquellos que no muestran ninguna relación entre variables. En conclusión, se corrobora que no existe una evidencia clara y generalizada que confirme la existencia de la metáfora de la pirámide deportiva en España.

PALABRAS CLAVE: Metáfora de la pirámide deportiva; Trickle up; trickle down; deporte de élite; deporte base

\section{ABSTRACT}

The sports pyramid metaphor (trickle down and trickle up effect) has been used by several governments to justify their public investments in elite sport. The scientific evidence of this effect is fragmented, previous studies question their veracity. This study examines the effect of the sports pyramid metaphor in Spain.

Using Spearman's correlation coefficient $(\rho)$, we tested for possible relationships between sporting success and the evolution of the federation membership history of 23 sports modalities during 1992-2016. According to results, three different groups of sports could be identified: 1) those that could be assumed to present both trickle down and up effects; 2) those with negative correlations between sporting success and membership figures; and 3) sports that do not show any relationships between those variables. As a conclusion, it is stated that there is no clear and generalized evidence to confirm the existence of the sport pyramid metaphor in Spain.

KEYWORDS: Sport pyramid metaphor; trickle up; trickle down; elite sport; mass participation.

\section{INTRODUCCIÓN}

Las administraciones públicas (AAPP) estatales, autonómicas y municipales, destinan parte de su presupuesto anual al deporte de élite. Dicha cantidad es imposible de cuantificar puesto que en los respectivos presupuestos no existen partidas económicas específicas en deporte de élite. La inversión de las AAPP en el deporte de élite se realiza mediante diferentes conceptos, unos con repercusión directa mientras que otros la tienen de forma indirecta. Así, para conocer la inversión pública en el deporte de élite, deberíamos atender las cantidades destinadas a:

1) repercusión directa: a) a las federaciones deportivas (FD), estatales y autonómicas. Así, por ejemplo, el Consejo Superior de Deportes (CSD), destinó $46 \mathrm{M} €$ en subvenciones a la alta competición durante el año 2017; b) a los centros de alto rendimiento y los centros de perfeccionamiento, con cantidades no publicadas; y c) ayudas a los deportistas olímpicos a través del plan ADO, promovido por el Gobierno a través del CSD (quien aporta parte del presupuesto) y con la participación de Radio Televisión Española, (quien posibilita la 
retransmisión de reportajes y/o competiciones a costa del presupuesto público), ambas cantidades no publicadas.

2) repercusión indirecta: a) la exoneración de tributación del Impuesto de la Renta de la Personas Físicas de los deportistas de alto nivel (competencia estatal) y deportistas de alto rendimiento (competencia autonómica), cantidad no cuantificada públicamente; b) la reducción de tributación del Impuesto de Sociedades de aquellas sociedades que patrocinen eventos deportivos considerados por el Estado de interés general (cantidad no cuantificada públicamente); c) la aportación de los beneficios de Loterías del Estado para determinados eventos; por ejemplo en el caso de los Juegos del Mediterráneo de Almería 2005 fue de 4,7M€; d) ayudas directas (inversión) para la organización de eventos deportivos internacionales, como por ejemplo los Juegos del Mediterráneo de Tarragona 2018, con 9M€ procedentes del Estado y $14 \mathrm{M} €$ de la CA de Catalunya; en los Juegos del Mediterráneo, Almería 2005, la cantidad ascendió a $40 \mathrm{M} €$ provenientes del Estado; e) la lucha contra el dopaje, a través de la AEPSAD; f) el gasto de los cuerpos nacionales y autonómicos para garantizar la seguridad y el orden en eventos deportivos; y g) la cantidad abonada por Radio Televisión Española en concepto de derechos para la retransmisión de competiciones deportivas.

Las AAPP españolas justifican el gasto público en el deporte de élite con el argumento que el éxito deportivo generará efectos positivos en la población (efecto trickle down): consolida y refuerza la identidad nacional (patriotismo), mejora el reconocimiento diplomático (prestigio internacional), desarrolla el deporte base (talentos) y amplia el deporte base (licencias deportivas y hábitos deportivos; Houlihan y Green, 2008; van Bottenburg et al., 2011; De Croock et al., 2012). Sin embargo, aun siendo un discurso muy utilizado, no existen estudios científicos, internacionales o nacionales, que sustenten o contradigan dicha afirmación (De Bosscher, Sotiriadou y Van Bottenburg, 2014).

Así, las AAPP utilizan el efecto trickle down como coartada muy posiblemente porque se da por hecho que el efecto es real y evidente $y$, por lo tanto, no resulta necesario demostrarlo (Grix y Carmichael, 2012). Pero si verdaderamente nos cuestionamos por qué las AAPP deben destinar fondos públicos al deporte de élite, seguimos sin una respuesta justificada (Coalter, 2007). Es por ello que cada vez son más las voces que cuestionan dicha política en pro de un gasto dirigido a la población general, al deporte para todos y deporte base (Van Bottenburg, 2003; Houlihan y Green, 2008). El objetivo de este trabajo es el de analizar la existencia de la metáfora de la pirámide deportiva en España y de los efectos trickle down o up.

\section{MARCO TEÓRICO}

\subsection{DEFINICIÓN DE CONCEPTOS BÁSICOS}

El objeto de este artículo pivota sobre algunos conceptos (deporte de alta competición, deporte de élite, deporte de alto nivel, deporte de alto rendimiento, deporte de masas, deporte para todos, deporte base y práctica deportiva) que, 
básicamente, presentan dos problemáticas: 1) terminología, muchos de ellos carecen de una definición clara e inequívoca en todos los ámbitos científicos: jurídico, académico, deportivo, educativo, administrativo, lo que genera conflictos y habitualmente son utilizados como sinónimos; y 2) cuantificación, resulta muy difícil cuantificar, mediante datos objetivos, la capacidad de cada tipo de deporte. Así, para evitar confusiones, definimos los dos conceptos básicos objeto de este artículo:

\section{DEPORTE DE ALTA COMPETICIÓN, DEPORTE DE ÉLITE O DEPORTE DE ALTO NIVEL Y ALTO RENDIMIENTO}

Son términos jurídicamente indeterminados. Sí existen las definiciones jurídicas de deportista de alto nivel, deportista de alto rendimiento (Real Decreto 971/2007) y deportista profesional (Real Decreto 1006/1985), pero no existe una definición jurídica de deporte de alta competición, élite o alto nivel. Desde un punto de vista administrativo, se considera "el deporte de alto nivel de interés para el Estado, en tanto que constituye un factor esencial en el desarrollo deportivo, por el estímulo que supone para el fomento del deporte base, en virtud de las exigencias técnicas y científicas de su preparación, y por su función representativa de España en las pruebas o competiciones deportivas oficiales de carácter internacional" (Ley 10/1990 del Deporte, art. 6). Desde un punto de vista académico, los tres conceptos se utilizan como sinónimos. En España, el desarrollo del deporte de alto nivel y alto rendimiento reside en las FD en colaboración con las administraciones públicas. Está financiado principalmente por el Estado.

A efectos del presente artículo, utilizaremos el término deporte de élite como genérico de deporte de alta competición o deporte de alto nivel y rendimiento y se entenderá por aquella práctica deportiva institucionalizada (existe competición reglada, récords y ránquines), con efecto internacional (reconocimiento), con independencia de la naturaleza jurídica de la organización (federada o no) y de los deportistas (profesionales o amateur), el objetivo de la cual es la especialización deportiva y la obtención del máximo rendimiento. Normalmente, se cuantifica el éxito del deporte de élite por número de medallas.

\section{DEPORTE BASE}

También se trata de un término jurídicamente indeterminado. A efectos de este artículo, se entiende por deporte base la actividad deportiva practicada con el objetivo de iniciarse o formarse en una modalidad deportiva e incluso la práctica del ocio, con independencia de la edad del practicante. Puede, o no, implicar la participación en competición, oficial o no, siendo el objetivo de ésta la formación, técnica, táctica y/o estratégica, y no la mejora del rendimiento y/o la especialización deportiva. En la mayoría de sistemas deportivos, es el paso previo al deporte de élite y es el que nutre a este último. A los efectos de este artículo, se cuantificará en número de licencias deportivas. 


\subsection{LA METÁFORA DE LA PIRÁMIDE DEPORTIVA}

La relación existente entre el deporte de élite y el deporte base, históricamente, ha sido vinculada mediante la metáfora de la pirámide deportiva. La metáfora de la pirámide deportiva interrelaciona el deporte de élite con el deporte base mediante una relación dependiente del uno con el otro. Así, cuantos más practicantes haya (deporte base) mayor será el éxito del deporte de élite, puesto que el primero nutrirá al segundo (efecto trickle up). A su vez, los éxitos logrados por el deporte de élite servirán de inspiración para que nuevos practicantes se inicien en el deporte base (efecto trickle down).

Del análisis de la literatura académica internacional se observa que es contradictoria y sigue sin aportar evidencias claras que justifiquen la relación entre el deporte de élite y el deporte base (Heinilä, 1982; Renson, 2002; van Bottenburg, 2003; Green 2005; Sotiriadou et al., 2008; Sotiriadou y Shilbury, 2009; Grix y Carmichael, 2012). La metáfora de la pirámide deportiva ha sido criticada porque, por un lado, mucha gente practica deporte sin ningún deseo de competir en élite (Eichberg et al., 1998); y, por el otro, el deporte de élite no se puede relacionar como una simple extensión del deporte base porque es posible construir un sistema deportivo de élite sin disponer de base deportiva (van Bottenburg, 2003; Green, 2005; De Bosscher y van Bottenburg, 2011). Deporte de élite y deporte base pueden evolucionar independientemente y la conexión entre ellos es compleja. Esta divergencia permite cuestionar cualquier política pública que utilice la metáfora de la pirámide deportiva para legitimar sus inversiones de gasto público (De Bosscher et al., 2008b). En el caso de la literatura académica nacional, es del todo inexistente, así no existen estudios previos que intenten analizar la metáfora de la pirámide deportiva.

\subsection{LA BÚSQUEDA DE EVIDENCIAS: EL EFECTO TRICKLE DOWN}

El efecto trickle down explica que el éxito del deporte de élite sirve de inspiración a los deportistas jóvenes (van Bottenburg, 2001) y se basa en la asunción de que existe la evidencia de dicho efecto sin ser, realmente, demostrada (Weed, 2009). Así, la asunción del efecto trickle down implica que el éxito del deporte de élite tiene una función inspiradora mediante: a) el rendimiento del deporte de élite; b) los deportistas de élite (como estrellas o figuras mediáticas); y c) el legado de los eventos deportivos.

Estos elementos (factores independientes) pueden afectar al número de practicantes (factor dependiente). Los efectos de los factores independientes pueden examinarse en relación a cuatro áreas: a) efectos en la participación deportiva: la atracción (captación de nuevos participantes), la retención (motivación o fidelización para continuar con la práctica deportiva), la motivación (avanzar hacia niveles más altos de la competición) y la base deportiva (asociado a incrementar el compromiso para avanzar en la élite deportiva); b) efectos de la elección de la modalidad deportiva; c) efectos en el comportamiento deportivo (i.e.; la elección de una determinada posición en deportes colectivos, la inspiración para copiar técnicas o tácticas deportivas, o incluso malas prácticas); y d) efectos en el comportamiento externo al deporte (i.e.; vestimenta, cortes de 
pelo, preferencias musicales, valores vinculados a consumo, o no, de drogas, racismo o género).

Siguiendo esta estructuración y tras la revisión de referencias bibliográficas se hace evidente que las investigaciones, hasta la fecha, a menudo se limitan a la literatura "gris" (conferencias, revistas no científicas, divulgación de opinión sin estar sujeta a revisión externa; Grix y Carmichael, 2012). Realizar un estudio empírico con el objetivo de determinar la metáfora de la pirámide deportiva no es sencillo ya que resulta muy difícil comprobar, filtrar y aislar las variables que intervienen (Wicker et al., 2012). Así, la variabilidad en la participación puede explicarse por muchos condicionantes a corto plazo (Sotiriadou y Shilbury, 2009; Grix y Carmichael, 2012): a) cambios en la disponibilidad de infraestructura deportiva: la natación española ha tenido éxito cuando en España se han construido piscinas climatizadas; b) la vida en un vecindario propenso a una actividad concreta: la ciudad de Terrassa aporta el $80 \%$ de los jugadores de la selección española de hockey hierba; Terrassa es la cuna del hockey hierba español, allí residen 4 clubes centenarios que vieron su apogeo en la celebración de los Juegos Olímpicos (JJOO) de Barcelona, dónde Terrassa fue sede deportiva de dicha modalidad. Así, en Terrassa, ser jugador de hockey hierba socialmente está mejor reconocido que ser futbolista; c) el efecto de los compañeros: el éxito del waterpolo femenino español puede explicarse por la determinación de un grupo nacido de los JJOO de Barcelona; d) el estado de un deporte en una escuela: el éxito del bádminton español nace de los programas escolares; y e) actividades organizadas para atraer a los jóvenes o incluso aumentar los ingresos o el Producto Interior Bruto: la organización de pruebas de trail running o de triatlón como estrategia para el desarrollo del turismo territorial. Teniendo en cuenta la complicada influencia de todos estos condicionantes, la investigación realizada hasta ahora no ha logrado encontrar una evidencia clara de que el éxito del deporte de élite tenga un efecto sobre el deporte base (efecto trickle down; Misener et al., 2015; Storm y Asserhoi, 2016).

A continuación, presentamos la discusión sobre la evidencia de los impactos de los tres factores independientes.

\section{LA FUNCIÓN INSPIRADORA DEL DEPORTE DE ÉLITE}

En los últimos años se han publicado algunos estudios en las áreas de política y gestión mediante el análisis de correlación o regresión para determinar la relación entre el éxito deportivo y las licencias deportivas principalmente en un único deporte (Frick y Wicker, 2016; Haut y Gaum, 2017). Los resultados de los estudios publicados son controvertidos; así varios investigadores identifican contra efectos en algunos deportes. Estos son deportes que han tenido éxito con el tiempo o han tenido deportistas relevantes pero cuya evolución de licencias deportivas ha disminuido (Stokvis y Minnee, 1986; van Bottenburg, 2003; Steward y Nicholson, 2004; Green, 2005; Feddersen et al., 2009). Este fue, por ejemplo, el caso del tenis en Alemania después del periodo de éxito de Boris Becker y Steffi Graff, o del voleibol en los Países Bajos después de ganar la medalla de oro en los JJOO de Atlanta 1996. Steward y Nicholson (2004) no encontraron ninguna correlación positiva significativa entre el éxito de la élite y la evolución en número de licencias deportivas en seis deportes en Australia 
durante un periodo de 14 años. Por el contrario, otros estudios han encontrado relaciones positivas entre el éxito deportivo y el incremento de licencias deportivas. Por ejemplo, Hanstad y Skille (2010) encontraron correlaciones positivas entre la evolución en licencias y las medallas conseguidas en JJOO y mundiales $(r=0,597 ; p<0,05)$ y los primeros 15 lugares $(r=0,690 ; p<0,05)$ en modalidades deportivas como el esquí de fondo y el tiro en Noruega. Un estudio de De Bosscher y De Knop (2003) revela que el éxito en el tenis en 43 naciones europeas estaba altamente correlacionado con el número de tenistas afiliados ( $r$ $=0,724 ; p<0,001)$ y el número de pistas de tenis $(r=0,858 ; p<0,001)$. Finalmente, van Bottenburg $(2002 ; 2003)$ encontró una correlación significativa entre el porcentaje de la población que participa solo en un deporte organizado y el número de medallas por millón de habitantes en 20 estados europeos $(r=0,535$; $p<0,01)$. Curiosamente, esta correlación se acrecentaba en aumentar la intensidad y la competitividad.

Si consideramos los estudios expuestos anteriormente de forma colectiva, estos sugieren la inexistencia de una evidencia clara del efecto trickle down, 0 simplemente la inexistencia de dicho efecto. Los resultados sugieren que, si existe una relación, puede ser específica para el deporte, el país y el contexto. Weed (2009), en su estudio con 1509 participantes de rugby y cricket en Nueva Zelanda, concluye que el efecto de modelo como consecuencia del éxito del deporte de élite no tiene un impacto significativo en aquellos que rara vez o nunca han participado $y$, por lo tanto, ya no están emocionalmente comprometidos con el deporte. Weed descubrió que este efecto sí afectaba a los que ya participaban en el deporte analizado, de tres maneras: 1) fomentaba una nueva participación de los participantes caducos -que se habían retirado-; 2) provocaba un aumento en la frecuencia de participación entre los participantes actuales; y 3) entre los participantes actuales, aumentaba la probabilidad de cambiar de actividad. Además, el efecto del modelo puede verse influido, entre otras, por otras variables como la cobertura de los medios de comunicación, la financiación, y los programas de participación masiva (van Bottenburg 2003, Feddersen et al. 2009).

En consecuencia, la naturaleza de la relación es complicada y los factores son difíciles de analizar. Por lo tanto, las preguntas sobre los mecanismos que afectan al incremento del deporte base siguen sin respuesta.

\section{LA FUNCIÓN INSPIRADORA DEL DEPORTE DE ÉLITE (DEPORTISTAS COMO ESTRELLAS)}

Una de las explicaciones más utilizadas y conocidas sobre el aumento de la popularidad de determinadas modalidades deportivas es que la participación se impulsa en respuestas a las victorias de campeones que captan la imaginación del público (van Bottenburg 2001). Esta área de investigación se relaciona con los efectos de los modelos a seguir. La literatura en esta área está mucho más desarrollada y se origina principalmente en la investigación de la psicología deportiva, ya sea en el contexto de individuos de logros sobresalientes ( $p$. ej.: Lockwood y Kunda, 1997) o en el contexto de personas que tienen cualidades que nos gustaría poseer (p. ej.: Guiliano et al., 2007). Esta literatura ofrece ideas sobre qué modelos de rol escogen los jóvenes, por qué los eligen, si estos 
modelos influyen en el comportamiento de los jóvenes y, de ser así, cómo. En consecuencia, padres, hermanos, profesores, entrenadores, estrellas del pop y héroes deportivos han sido estudiados como modelos en varios contextos: teorías de aprendizaje social (Bandura 1977; Jung, 1986, Lockwood y Kunda, 1997), estudios de comportamiento dentro y fuera del deporte (Biskup y Pfister, 1999; Carr y Weigand, 2001; Vescio et al., 2005; Guiliano et al., 2007; Lines 2011), sociología (Fleming et al., 2005; Buford 2009), pedagogía (Bromnick y Swallow, 1999) y educación (Nauta y Kokaly, 2001). Un hallazgo interesante de Lockwood y Kunda (1997) es que las superestrellas, como modelos a seguir, provocan inspiración y autoevaluación cuando su éxito parece alcanzable para el observador, pero provocan la autodeflación cuando parece inalcanzable. Además, varios autores han encontrado que los varones son más propensos a elegir un deportista como modelo a seguir que las niñas (por ejemplo, Ewens y Lashuk, 1989; Biskup y Pfister, 1999; Bromnick y Swallow, 1999; Jones y Schumann, 2000; Guiliano et al., 2007; Lines 2011). Algunos autores también destacan los posibles efectos negativos de los héroes como modelos (por ejemplo, Hindson et al., 1994; Globus, 1998; Payne et al., 2003; Lines 2011) y cómo los medios de comunicación transmiten y amplían el impacto de los modelos de rol para los jóvenes (French y Pena, 1991; Biskup y Pfister, 1999; Fleming et al., 2005; Lines 2011).

Si bien esta literatura proporciona información sobre los efectos de los modelos a seguir, muy pocas investigaciones han examinado el efecto de los deportistas de élite como modelos a seguir en la participación deportiva o desarrollo deportivo (Green 2005; Sotiriadou et al., 2008).

\section{LA FUNCIÓN INSPIRADORA Y EL LEGADO DE LOS EVENTOS DEPORTIVOS DE ÉLITE}

El legado de un mega evento deportivo se ha convertido en un resultado que se presume que se produce $y$, además, ha tenido un impacto en cómo las organizaciones los planifican e implementan (Leopkey y Parent, 2012). A pesar de los beneficios potencialmente comprobables de organizar un mega evento deportivo, no existe un método fiable y ampliamente aceptado para evaluar sus efectos (London East Research Institute, 2007; Veal et al., 2012). Por un lado, muchos son los autores que cuestionan que los grandes eventos deportivos realmente generen un impacto positivo en la participación deportiva (Coalter, 2007; EdComs, 2007; Girginov y Hills, 2008; Veal et al., 2012); mientras que, por otro lado, son pocos los estudios que muestran efectos positivos y estos son, predominantemente, a corto plazo (Hanstad y Skille, 2010). Por ejemplo, Frawley y Crush (2011) y Veal et al. (2012) determinaron que hubo un crecimiento acelerado del número de jugadores de rugby en Australia dos años después de la Copa del Mundo de 2003.

Finalmente, se puede concluir que, en términos generales, todavía no existen evidencias claras de las consecuencias del efecto trickle down del éxito del deporte de élite, los deportistas de élite y los eventos deportivos, y que es especialmente difícil determinar una relación causa/efecto. 
Así, el presente estudio examina la relación bidireccional entre el éxito del deporte de élite y el deporte base, evaluando, así, la existencia de los efectos trickle down y up. El artículo constituye la primera aportación que se hace en España y, a su vez, incrementará el conocimiento supraestatal en relación a la metáfora de la pirámide deportiva.

\section{MÉTODO}

\subsection{RECOGIDA DE DATOS}

Se analizan 23 modalidades deportivas que han obtenido, como mínimo, una medalla en campeonatos mundiales o JJOO celebrados en el periodo 19922016.

Se identifica el éxito del deporte de élite utilizando el Índice de Deporte de Élite (ESI, por sus siglas en inglés) similar al utilizado en De Bosscher et al. (2013). EI ESI se calcula a partir de adjudicar 10 puntos a la medalla de oro, 8 a la de plata y 6 a la de bronce. Asimismo, siguiendo el razonamiento de investigaciones anteriores (De Bosscher et al., 2008, 2013) se multiplican estos valores por 6 (JJOO) o por 4 (campeonatos mundiales). De la suma de estas puntuaciones se obtiene una calificación total de éxito deportivo por año y modalidad deportiva. Cabe tener en cuenta que la celebración de campeonatos mundiales no es homogénea entre modalidades deportivas (i.e.; algunos son anuales -ciclismo-, otros bianuales -atletismo, tiro con arco- y otros bienales -futbol, baloncesto-), por ello se adapta el cálculo ESI a cada modalidad deportiva teniendo en cuenta la puntuación obtenida en un campeonato hasta la celebración de la nueva edición. También hay que señalar que, en el caso del tenis, y a los efectos de este estudio, se contabilizan los Grand Slam como campeonatos del mundo.

Para identificar el deporte base, a los efectos de este artículo se analiza el número de licencias deportivas de cada modalidad deportiva analizada. El número de licencias se obtiene a partir de los datos oficiales publicados por el CSD en su página web (http://www.csd.gob.es/). Se excluyen aquellas modalidades en que la tipología de licencia deportiva no se corresponde con la tipología de competición (por ejemplo, la natación. Existe una licencia deportiva única con diferentes competiciones: natación, waterpolo, natación sincronizada o saltos).

\subsection{ANÁLISIS DE DATOS}

El planteamiento del análisis de datos se basó en el desarrollado en investigaciones previas (e.g.; De Bosscher et al., 2013). En primer lugar, se realiza un análisis descriptivo de las puntuaciones ESI y el número de licencias, mediante el cálculo de la media de ambas variables para cada deporte en el periodo 1992-2016. A continuación, se calculan las correlaciones de Spearman ( $\rho)$ entre las puntuaciones ESI y el número de licencias para cada deporte. Se utiliza el coeficiente de correlación de Spearman dada la naturaleza no paramétrica de los datos del estudio. La expresión matemática de este coeficiente es la siguiente: $\rho=1-\frac{6 \sum d_{i}^{2}}{n\left(n^{2}-1\right)}$. 
Teniendo en cuenta que se esperaba que los efectos no se produjeran en el mismo año que se celebraba un acontecimiento, sino en los años anteriores (trickle up) o posteriores (trickle down), las puntuaciones ESI se correlacionaron con el número de licencias de ese año $(X)$, de los años anteriores (i.e.; $X-1, X-2$, $X-3, X-4)$ y de los años posteriores (i.e.; $X+1, X+2, X+3, X+5, X+8, X+10$ ). Con $X$ nos referimos a cualquier año dentro del rango de años analizados en el presente estudio (1992-2016). Las correlaciones se calculan únicamente para aquellos años en que se dispone de datos. Estas correlaciones se plantean también entre las puntuaciones ESI y el incremento/decremento anual en el número de licencias. En todos los análisis de correlación se utiliza un nivel de significación de .05. Los coeficientes de correlación han sido interpretados siguiendo el criterio de Safrit y Wood (1995), según el cual éstos se clasifican en ausencia de correlación $(0-, 19)$, correlaciones bajas $(, 20-, 39)$, correlaciones moderadas $(, 40-, 59)$, correlaciones moderadamente altas $(, 60-, 79)$, y correlaciones altas $(, 80)$. Todos los análisis de datos fueron efectuados con el programario estadístico PASW Statistics v. 17.

\section{RESULTADOS}

\subsection{DATOS DESCRIPTIVOS DE LAS PUNTUACIONES ESI Y EL NÚMERO DE LICENCIAS POR DEPORTE}

Las Figuras 1 y 2 ilustran una visión general del deporte de élite en España durante el periodo 1992-2016. Por un lado, la Figura 1 presenta las puntuaciones ESI medias para cada uno de los 23 deportes durante ese periodo. Puede observarse que deportes como el ciclismo, la vela, el taekwondo y el piragüismo muestran los mejores resultados deportivos y, por el contrario, el voleibol, la halterofilia, la lucha olímpica y el remo tuvieron escaso éxito. La media ESI para el deporte español en el periodo 1992-2016 fue de 66,42 puntos ( $D T=72,70)$. Por otro lado, la Figura 2 presenta el número medio de licencias por deporte durante el periodo 1992-2016. Así el fútbol destaca como deporte mayoritario, seguido del baloncesto y el judo. Por el contrario, la halterofilia y el boxeo son los deportes minoritarios. La media de licencias por año y deporte durante el periodo de estudio fue de 75000 licencias $(D T=148188,09)$. 
Figura 1. Puntuaciones medias españolas del índice de deporte de élite (ESI) para cada deporte durante el periodo 1992-2016. La línea en naranja marca la puntuación media ESI para ese periodo $(66,42$ puntos; $D T=72,70)$.

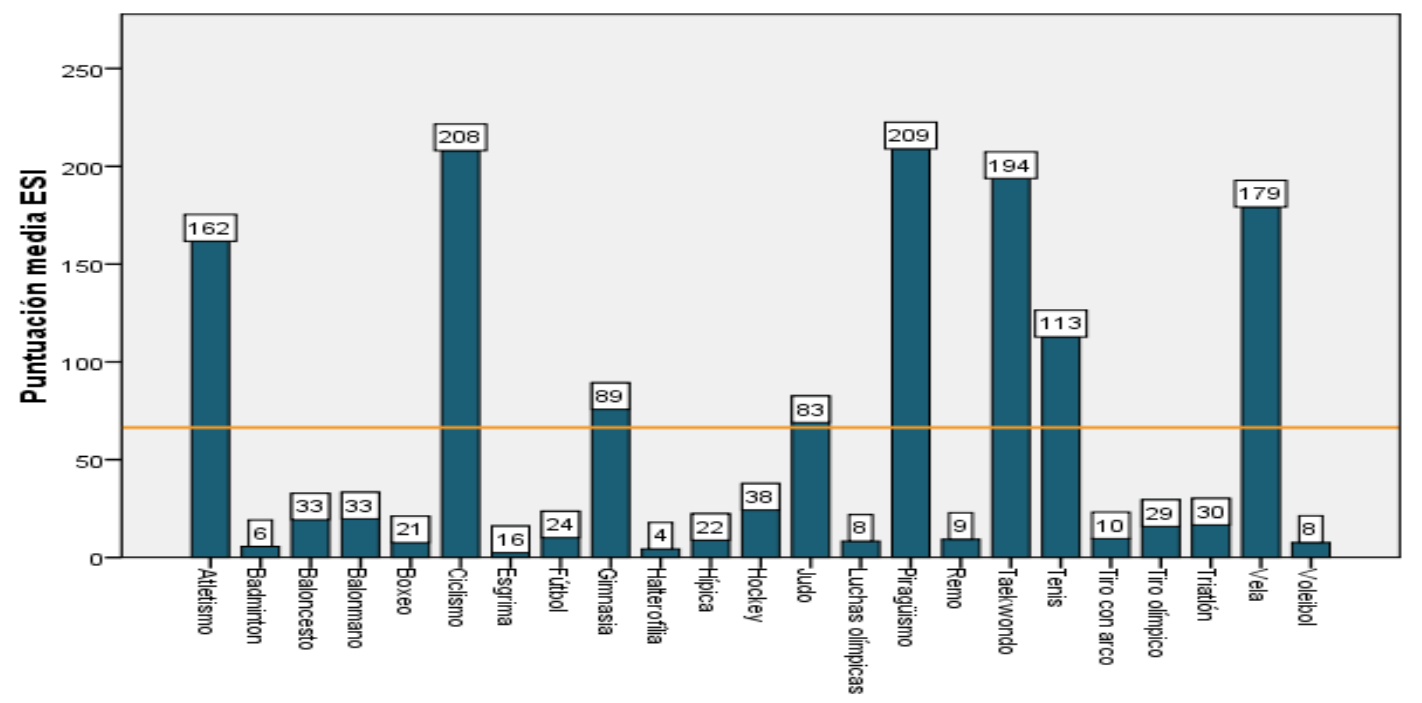

Figura 2. Número de licencias promedio por deporte en España para el periodo 1992-2016. La línea en naranja marca la media de licencias por deporte durante ese periodo (75000; $D T=$ 148188,09)

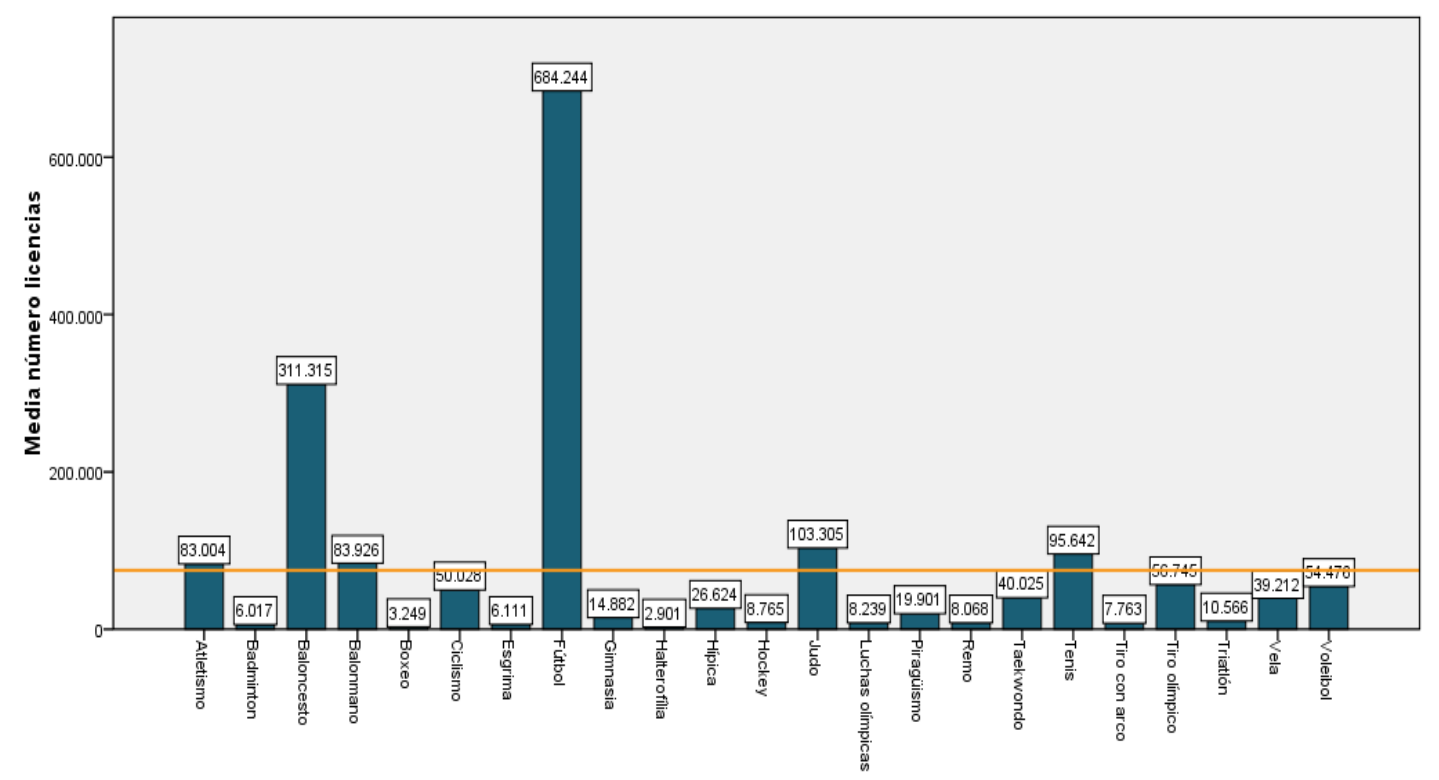

\subsection{CORRELACIONES ENTRE PUNTUACIONES ESI Y EL NÚMERO DE LICENCIAS EN ESPAÑA}

La Tabla 1 presenta las correlaciones entre las puntuaciones ESI y el número de licencias, tanto para el año en cuestión como para los años anteriores (efecto trickle up) y posteriores (efecto trickle down). Como puede observarse, la mayoría de los deportes exhiben correlaciones estadísticamente significativas, como mínimo para una de las relaciones analizadas. Es interesante señalar que dichas correlaciones pueden ser tanto positivas (i.e.; el comportamiento de las puntuaciones ESI y el número de licencias van en la misma dirección) como negativas (i.e.; el comportamiento de las puntuaciones ESI y el número de 
licencias van en direcciones opuestas). Así, por ejemplo, el baloncesto muestra correlaciones altas y positivas entre ambas variables en distintos momentos temporales, manifestando tanto un efecto trickle up como trickle down. En cambio, la gimnasia muestra correlaciones moderadamente altas y negativas entre las puntuaciones ESI y el número de licencias, indicando que ambas variables se relacionan de forma inversa (i.e.; los momentos de éxito deportivo se vinculan con años con menor número de licencias, y viceversa). Con el propósito de identificar de qué distintas maneras pueden relacionarse las puntuaciones ESI con el número de licencias, en el apartado siguiente se agrupan los 23 deportes en función del comportamiento de las correlaciones presentadas.

Tabla 1. Coeficientes de correlación Spearman entre el éxito deportivo (ESI) en un año X (cualquiera dentro del rango 1992-2016) y las licencias en ese año $X$, en los años anteriores $X$ $1, \mathrm{X}-2, \mathrm{X}-3, \mathrm{X}-4$ (trickle up) y en los años posteriores $\mathrm{X}+1, \mathrm{X}+2, \mathrm{X}+3, \mathrm{X}+5, \mathrm{X}+8, \mathrm{X}+$

\begin{tabular}{|c|c|c|c|c|c|c|c|c|c|c|c|}
\hline & $\mathbf{X}$ & $X-4$ & $X-3$ & $X-2$ & $X-1$ & $X+1$ & $X+2$ & $X+3$ & $X+5$ & $X+8$ & $X+10$ \\
\hline Atletismo &, $590^{* \star}$ & ,189 & ,342 & ,393 &, $518^{\star \star}$ &, $573^{\star \star}$ &, $564^{\star \star}$ & , 152 & , 120 & , 194 & ,238 \\
\hline Bádminton &, $397^{*}$ & ,337 & ,288 & ,243 & ,313 & ,152 & ,022 & & & & $\mathrm{a}$ \\
\hline Baloncesto &, $821^{* *}$ &, $858^{* *}$ &, $853^{* *}$ & ,832** &, $822^{* *}$ &, $833^{* *}$ &, $831^{* *}$ &, $787^{* *}$ &, $561^{*}$ & ,089 &,- 062 \\
\hline Balonmano &, $430^{*}$ &,- 027 & 156 & ,362 & , $535^{\star \star}$ & , $442^{*}$ & ,336 & ,304 &, $529^{*}$ & ,334 &, $586^{*}$ \\
\hline Boxeo &,- 052 &,- 028 &,- 077 &,- 053 & ,125 &,- 058 &,- 151 &,- 284 &,- 199 & 123 & 108 \\
\hline Ciclismo &,- 269 &,$- 486^{*}$ &,$- 477^{*}$ &,$- 410^{*}$ &,- 338 &,- 192 &,- 098 & ,032 & ,329 &, $871^{* *}$ &, $685^{* *}$ \\
\hline Esgrima & , 133 &,- 089 &,- 018 & ,053 & ,089 &,- 012 &,- 083 &,- 221 &,- 380 &,- 261 & ,013 \\
\hline Fútbol &,$- 481^{*}$ &,$- 446^{*}$ &,$- 460^{*}$ &,$- 512^{\star *}$ &,$- 484^{*}$ &,$- 457^{*}$ &,- 360 &,- 370 &,$- 457^{*}$ &,$- 630^{* *}$ &,$- 592^{*}$ \\
\hline Gimnasia &,$- 787^{\star \star}$ &,$- 581^{* *}$ &,$- 624^{* *}$ &,$- 658^{\star \star}$ &,$- 751^{\star *}$ &,$- 733^{\star *}$ &,$- 704^{\star *}$ &,$- 655^{\star \star}$ &,$- 557^{\star}$ &,- 135 &,- 379 \\
\hline Halterofilia & ,341 &,- 053 & , 196 & ,018 & 233 & ,305 &, $419^{*}$ & 361 & ,338 &,- 153 &,- 309 \\
\hline Hípica & ,004 &,$- 552^{\star *}$ &,$- 407^{*}$ &,- 237 &,- 094 & ,092 & 143 & 249 &, $565^{\star \star}$ & ,686** &, $531^{*}$ \\
\hline Hockey &,$- 410^{*}$ &,- 044 &,- 231 &,- 213 &,- 339 &,- 363 &,$- 443^{*}$ &,- 401 &,- 437 &,$- 744^{* *}$ &,$- 826^{* *}$ \\
\hline Judo &,$- 548^{* *}$ &,$- 646^{* *}$ &,$- 589^{* *}$ &,$- 570^{\star *}$ &,$- 681^{\star *}$ &,$- 471^{*}$ &,- 398 &,$- 453^{*}$ &,- 349 &, $531^{*}$ &, $585^{\star}$ \\
\hline & ,240 & ,483* & 282 & ,373 & 340 & 301 & ,308 & ,056 & ,219 & ,306 &,- 371 \\
\hline Piragüismo &,- 008 & 218 & 113 & ,001 &,- 053 & ,044 & ,094 & ,047 & 072 &,- 407 &,$- 681^{* *}$ \\
\hline Remo & ,002 &,- 130 &,- 164 &,- 188 &,- 118 &,- 009 & ,028 &,- 024 & ,044 & , 194 & 147 \\
\hline Taekwondo &,$- 405^{*}$ &,- 130 &,- 097 &,- 076 &,- 244 &,- 074 & ,272 &,- 116 & , 120 &,- 163 & ,350 \\
\hline Tenis &, $401^{*}$ & ,355 & ,455* & , 457* &, $456^{*}$ & ,396 & 169 &,- 165 &,$- 704^{\star *}$ &,$- 588^{*}$ &,- 046 \\
\hline $\begin{array}{l}\text { Tiro con } \\
\text { arco }\end{array}$ &,$- 560^{* *}$ &,- 030 &,- 151 &,- 348 &,$- 484^{*}$ &,$- 646^{* *}$ &,$- 657^{* *}$ &,$- 650^{* *}$ &,$- 694^{* *}$ &,$- 651^{\star *}$ &,- 384 \\
\hline $\begin{array}{l}\text { Tiro } \\
\text { Olímpico }\end{array}$ & 125 & 100 & 173 & ,056 & ,071 & 130 & ,251 & ,458* & 189 &,- 241 &,$- 563^{*}$ \\
\hline Triatlón &, $766^{\star \star}$ &, $720^{\star *}$ &, $754^{\star \star}$ & , 769** &, $764^{\star *}$ & , $740^{* *}$ &, $693^{\star *}$ &, $694^{* \star}$ & , 568 & , $641^{* *}$ & 449 \\
\hline Vela &,- 323 & -,294 &,- 065 &,- 180 & -,268 &,$- 503^{*}$ &,$- 544^{\star *}$ &,$- 514^{*}$ & -,056 &,- 052 &,- 047 \\
\hline Voleibol &,- 287 &,- 106 &,$- 424^{*}$ &,$- 454^{\star}$ &,- 393 &,- 129 & , 052 & ,074 & ,043 & 226 &, $694^{\star \star}$ \\
\hline
\end{tabular}

Nota. ${ }^{*} p \leq, 05,{ }^{* *} p \leq, 01 .{ }^{a}$ La correlación no pudo ser calculada por no hallarse éxito deportivo (i.e.; puntuación ESI distinta de cero). 


\subsection{ANÁLISIS DE LAS RELACIONES ENTRE PUNTUACIONES ESI Y NÚMERO DE LICENCIAS}

\subsubsection{DEPORTES QUE MUESTRAN EFECTOS TRICKLE UP Y TRICKLE DOWN}

El primero de los subgrupos incluye una serie de deportes que muestran correlaciones positivas y altas o moderadamente altas entre la evolución en el número de licencias y las puntuaciones ESI. En este grupo se incluyen el atletismo, el balonmano, el baloncesto y el triatlón, siendo los dos últimos los ejemplos más claros. En base a la Tabla 1, podría deducirse que las luchas olímpicas también podrían formar parte de este grupo dado que las correlaciones irían en la misma dirección, a pesar de ser menores y no estadísticamente significativas.

En este primer grupo se puede observar tanto el efecto trickle up para los años precedentes, como el efecto trickle down para los años posteriores a un año $\mathrm{X}$. Así, estos deportes se caracterizan por un incremento tanto en el número de licencias como en el éxito deportivo (i.e.; puntuación ESI). Por ejemplo, en la Figura 3 se presenta la evolución de las puntuaciones ESI y el número de licencias a lo largo de los años en el triatlón. Así, se advierte que el incremento en el número de licencias va de la mano de la época con mayor éxito deportivo (a partir de 2001). En el caso del triatlón, la correlación entre la puntuación ESI y el número de licencias en ese mismo año X fue de ,766 $(p \leq, 01)$.

Figura 3. Evolución del número de licencias y las puntuaciones ESI en el periodo 1992-2016 en el triatlón.

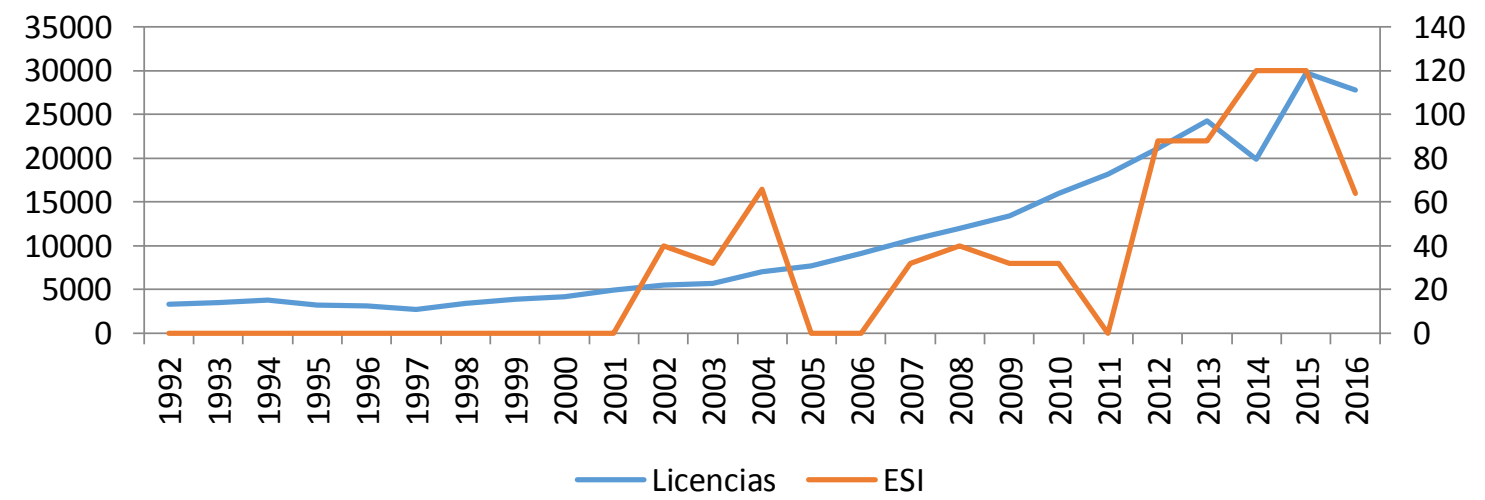

Un caso especial dentro de este grupo de deportes es el del tenis (ver Figura 4). Como puede observarse en la Tabla 1, este deporte exhibe solamente un efecto trickle up, dado que únicamente las correlaciones entre el número de licencias en los años precedentes y las puntuaciones ESI son positivas y muestran significación estadística. Este efecto se puede observar más fácilmente en los periodos 1992-1994 y 2002-2011, donde el incremento en el número de licencias precede al incremento del éxito deportivo. Además, el tenis muestra correlaciones negativas y significativas en los efectos a largo plazo (i.e.; $X+5 y$ $X+8$ ), seguramente explicadas por los descensos e incrementos en los éxitos deportivos que, 5 y 8 años después, coinciden con una evolución opuesta en el número de licencias. Por ejemplo, los descensos en el rendimiento deportivo de 
los periodos 1995-1997 y 1999-2000, van acompañados de un aumento en el número de licencias 5 y 8 años después. Por último, la halterofilia presenta únicamente un ligero efecto trickle down, siendo la correlación con el año X +2 la única que presenta significación estadística $(\rho=, 419$; ver Tabla 1).

Figura 4. Evolución del número de licencias y las puntuaciones ESI en el periodo 1992-2016 en el tenis.

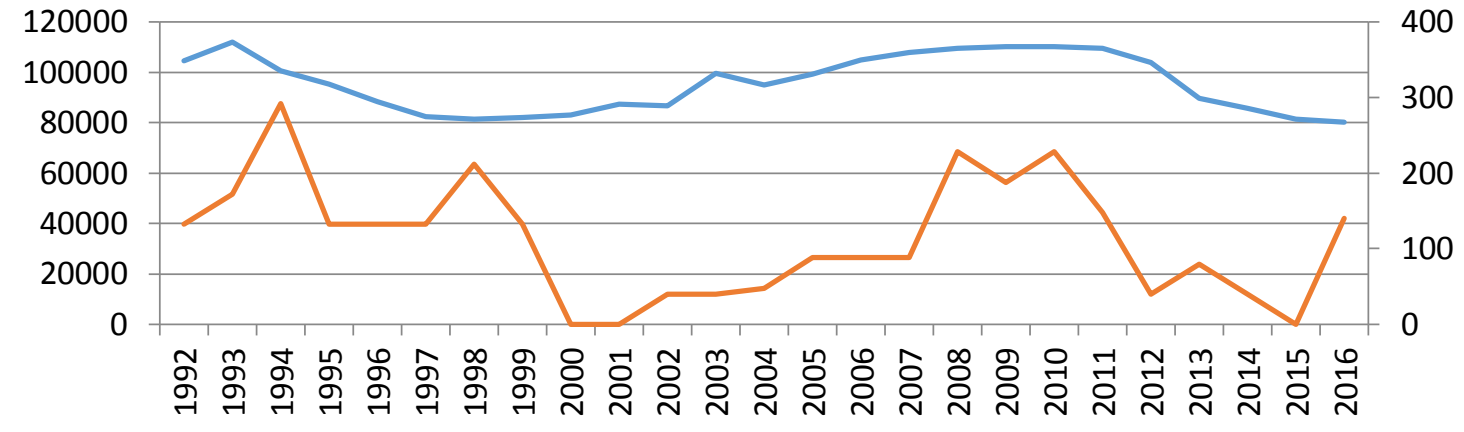

Licencias

\subsubsection{DEPORTES CON RELACIÓN NEGATIVA ENTRE LAS PUNTUACIONES ESI Y EL NÚMERO DE LICENCIAS}

Un segundo grupo de deportes incluye aquéllos con una correlación negativa entre el número de licencias y las puntuaciones ESI, tanto en los años anteriores al año $\mathrm{X}$ como en los posteriores. Este grupo incluye el fútbol, la gimnasia, el judo y el tiro con arco. En estos deportes, la evolución del número de practicantes y del éxito deportivo va en direcciones opuestas. Así, por ejemplo, un incremento en el número de licencias se relaciona con un menor éxito deportivo en los años posteriores, y un buen rendimiento deportivo en los años precedentes conlleva, paradójicamente, un descenso en el número de licencias de los años posteriores. En la Figura 4 se muestra la evolución del judo en el periodo 1992-2016. Como puede observarse, el número de licencias se incrementa ligeramente en este periodo, pero el éxito deportivo disminuye con el paso de los años. Asimismo, en los años 2008-2010, el número de licencias disminuye ligeramente, pero el éxito deportivo se incrementa. Complementariamente, el judo también muestra correlaciones moderadas y positivas a largo plazo $(\rho x+8=, 531$ y $\rho x+10=, 585$; $p<, 01)$. La correlación global entre la puntuación ESI y el número de licencias en ese mismo año fue de -,548 $(p<, 01)$. Finalmente, en este segundo grupo también hay que incluir el ciclismo, la hípica y el voleibol, que muestran correlaciones negativas entre el número de licencias y las puntuaciones ESI únicamente en los años precedentes a un año $\mathrm{X}$, y el hockey y la vela, que solamente las presentan en los años posteriores (ver Tabla 1). 
Figura 5. Evolución del número de licencias y las puntuaciones ESI en el periodo 1992-2016 en el judo.

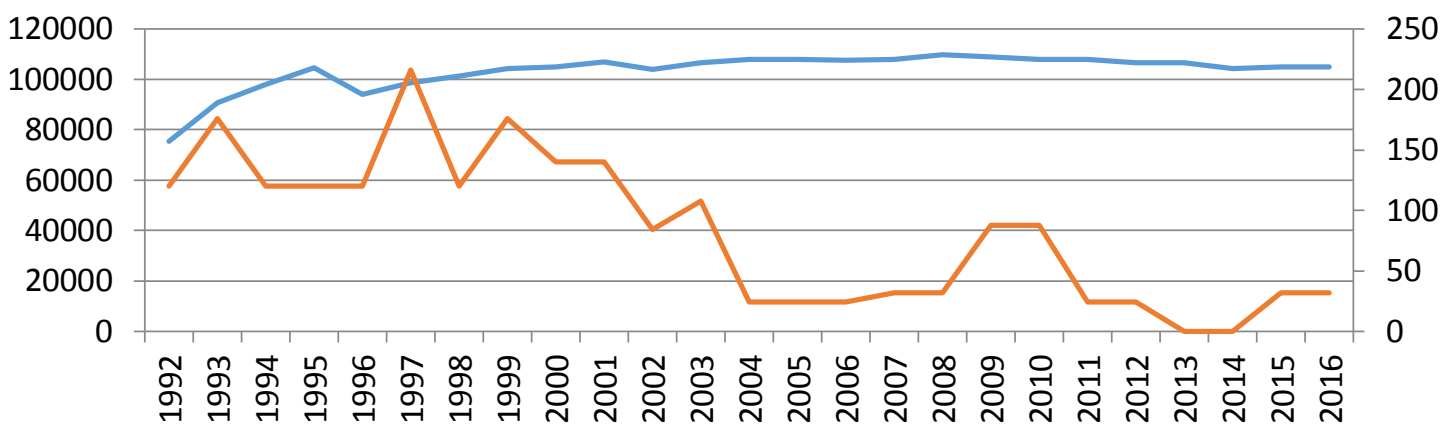

Licencias

\subsubsection{DEPORTES SIN RELACIÓN ENTRE LAS PUNTUACIONES ESI Y EL NÚMERO DE LICENCIAS}

El tercer y último grupo de deportes agrupa aquellas disciplinas sin correlaciones estadísticamente significativas entre las puntuaciones ESI y el número de licencias. Es decir, se trata de deportes cuyo éxito deportivo y cuya evolución en el número de licencias no están vinculados, incrementando o disminuyendo los valores de uno de ellos sin tener repercusión en el otro. En este grupo se incluyen el bádminton, el boxeo, la esgrima, el piragüismo, el remo, el taekwondo y el tiro con arco. A excepción del piragüismo y el taekwondo, estos deportes se caracterizan por haber obtenido un bajo éxito deportivo (ver Figura 1). Tal como puede observarse en la Tabla 1, los momentos puntuales de éxito deportivo no se relacionan con la evolución en el número de licencias. En la Figura 6 se presenta la evolución en las puntuaciones ESI y el número de licencias para el periodo 1992-2016 para el remo. Como puede observarse, los éxitos deportivos alcanzados en los años 1993, 2002 y 2006 no tienen demasiada repercusión en el número de licencias. Asimismo, el mayor incremento de licencias experimentado en los años 2012-2014 no tiene repercusión en la consecución futura de éxitos deportivos. La correlación global entre las puntuaciones ESI y las licencias de remo en un año X fue inexistente $(\rho=, 002 ; p>, 05)$.

Figura 6. Evolución del número de licencias y las puntuaciones ESI en el periodo 1992-2016 en el remo.

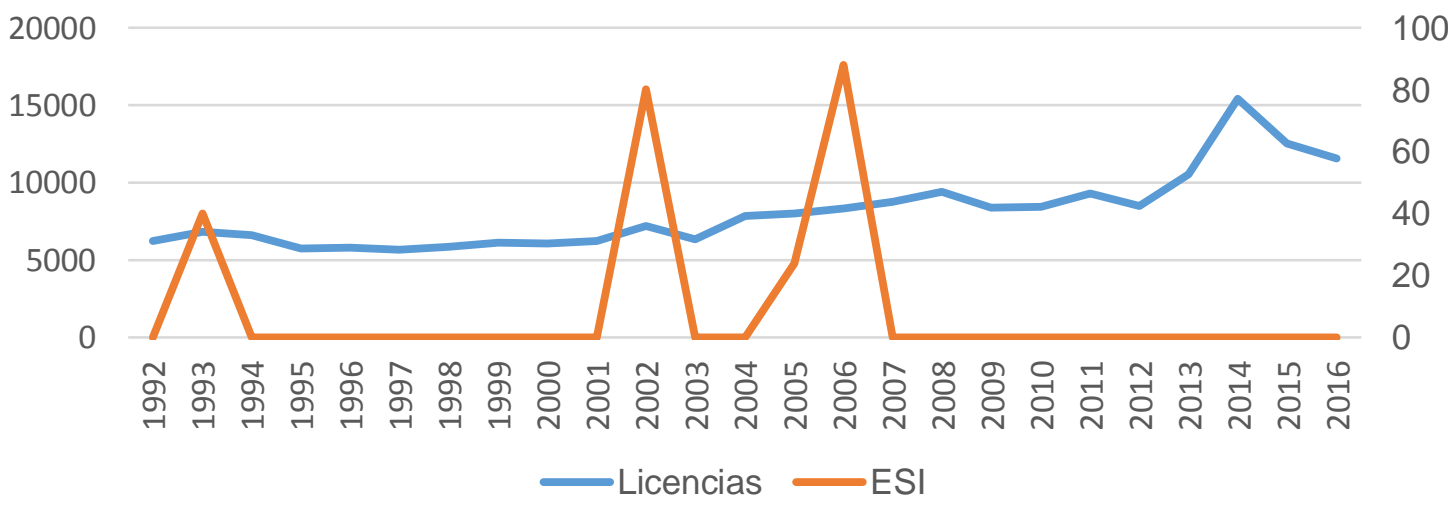




\section{DISCUSIÓN Y CONCLUSIONES}

El presente trabajo ha tratado de explorar la metáfora de la pirámide deportiva en el contexto del deporte español. Para ello se ha analizado la relación entre el éxito del deporte de élite y el deporte base, mediante el análisis de la correlación entre los resultados en competiciones internacionales y la evolución del número de licencias del deporte español entre los años 1992-2016 y, además, se asume que, si esta relación existiera, podría no materializarse inmediatamente y hacerlo hasta varios años después del éxito deportivo.

Tras el análisis estadístico de 23 modalidades deportivas en España se han identificado tres grupos: 1) un primer grupo formado por 4 modalidades deportivas (atletismo, balonmano, baloncesto y triatlón) con indicios estadísticos de la existencia del efecto trickle down y up; 2) un segundo grupo formado por 4 modalidades deportivas (gimnasia, judo, fútbol y tiro con arco) con correlaciones negativas entre el número de licencias y el éxito deportivo; y 3) un tercer grupo formado por el resto de modalidades deportivas y que no muestra relaciones entre el número de licencias y el éxito deportivo.

De esta forma, el presente estudio corrobora que, en España y para las modalidades deportivas analizadas, no existe una evidencia clara que confirme la existencia del efecto trickle down, tal y como apuntan estudios previos (van Bottenburg, 2002; De Bosscher y De Knop, 2003; Stewart y Nicholson, 2004; Feddersen et al., 2009; Hanstad y Skille, 2010; De Bosscher y van Bottenburg, 2011; De Bosscher et al., 2014). También apunta la dificultad de discernir tendencias generales, coincidiendo con la conclusión principal del estudio de De Bosscher et al. (2014). Y todo ello nos lleva a contradecir la afirmación, sin fundamento científico que, en muchas ocasiones, justifica la política e inversión de las AAPP sobre el deporte de élite y, por lo tanto, evidencia la necesidad de continuar con la exploración empírica la metáfora de la pirámide deportiva antes de afirmar su aceptación y existencia.

Sobre el estudio presentado cabe tener presente algunas limitaciones. En primer lugar, se identifica el deporte base mediante el número de licencias federativas publicadas por el CSD; siendo esta asunción no real puesto que existen muchos practicantes fuera del paraguas federativo. De este modo, las licencias federativas reflejan la participación en un entorno principalmente competitivo y, de acuerdo con van Bottenburg (2003), ofrecen una visión limitada de la realidad. En segundo lugar, el hecho de centrar el análisis en un único país, tal y como ocurre en la mayoría de estudios previos (i.e.; De Bosscher et al., 2011), reduce las posibilidades de generalizar los resultados y poder, así, confirmar la existencia de los efectos de trickle up y down. Por último, el hecho que exista correlación estadística no significa que haya relación de causalidad puesto que existen múltiples variables que pueden influir ineludiblemente sobre la posible relación entre el deporte de élite y el deporte base, que no se han contemplado en el presente estudio, y algunas de las cuales si han sido estudiadas en trabajos previos: 1) la cobertura de los medios de atletas y disciplinas deportivas (van Bottenburg 2003; Fleming et al., 2005; Sotiriadou y Shilbury, 2009; Hanstad y Skille, 2010; Lines, 2011); 2) La existencia de organizaciones, programas, concursos o instalaciones orientadas a la promoción deportiva de base (van 
Bottenburg 2002, Sotiriadou et al., 2008; Hanstad y Skille, 2010). Así, se recomienda que futuras investigaciones utilicen métodos estadísticos más avanzados, como los análisis con datos de panel (e.g.; Weimar, Wicker, y Prinz, 2015) o la regresión lineal (e.g.; Frick y Wicker, 2016), que permitan la inclusión de estas covariables en los análisis estadísticos.

Los resultados de este estudio permiten generar nuevas preguntas: ¿qué características tiene el atletismo, balonmano, baloncesto y triatlón que hacen que se produzca la metáfora de la pirámide deportiva?; ¿ por qué estudios parecidos (De Bosscher et al., 2014) realizados en otros territorios las modalidades que presentan correlaciones positivas son otras (atletismo, gimnasia, judo y tennis)?; ¿qué particularidades tienen la gimnasia, judo, futbol y tiro con arco que hace que tengan una correlación negativa en ambos sentidos? y ¿qué características tienen el resto de modalidades analizadas en las que no hay relación?

En conclusión, este artículo supone el primer estudio realizado en el contexto deportivo español que ha puesto a prueba la metáfora de la pirámide deportiva. Los hallazgos evidenciados son parciales y no muestran una evidencia definitiva de la metáfora de la pirámide deportiva. Pero si se reafirma la necesaria precaución a la hora de hacer afirmaciones definitivas sobre la metáfora de la pirámide deportiva, su existencia y sus efectos. Las instituciones y organizaciones deportivas nacionales deben ser conscientes de que su efecto no es un fenómeno evidente que fluye automáticamente del éxito deportivo y tampoco en todos los deportes.

\section{REFERENCIAS BIBLIOGRÁFICAS}

Bandura, A. (1977). Social learning theory. Englewood Cliffs, NJ: Prentice Hall.

Biskup, C. y Pfister, G. (1999). I would like to be like her/him: are athletes role-models for boys and girls? European physical education review, 5 (3), 199-218. DOI: https://doi.org/10.1177/1356336X990053003

Bromnick, R. y Swallow, B. (1999). I like being who I am: a study of young people's ideals. Educational studies, $25 \quad$ (2), 117-128. DOI: https://doi.org/10.1080/03055699997855

Buford May, R. (2009). The good and bad of it all: professional black male basketball players as role models for young black male basketball players. Sociology of sport journal, 3, 443-461. DOI: https://doi.org/10.1123/ssj.26.3.443

Carr, S. y Weigand, D. (2001). Parental, peer, teacher and sporting hero influence on the goal orientations of children in physical education. Physical education review, 7 (3), 305-328. DOI: https://doi.org/10.1177/1356336X010073005

Coalter, F. (2007). A wider social role for sport Who's keeping the score? London: Routledge.

De Bosscher, V. y De Knop, P. (2003). The influence of sports policies on international success: an international comparative study. In: NOC*NSF, ed. Proceedings of the 9th World Sport for All Congress, 'Sport for All and Elite Sport: Rivals or partners?' (Abstract). Ahrnem: NOC*NSF, 31.

De Bosscher, V., Sotiriadou, P. y Van Bottenburg, M. (2014). Scrutinizing the sport pyramid metaphor: an examination of the relationship between elite success and mass participation in Flanders. International Journal of Sport Policy and Politics, 5, 3: 319-339. DOI: https://doi.org/10.1080/19406940.2013.806340 
De Bosscher, V. y van Bottenburg, M. (2011). Elite for all, all for elite? An assessment of the impact of sport development on elit esport succés. En: Houlihan, B. Ed. Handbook on sport development. London: Routledge, 575-595.

De Bosscher, V., De Knop, P., and Truyens, J. (2008a). Hoe succesvol zijn we in topsport? De ontwikkeling van een Topsportindex. [How successful are we in elite sport? The development of an elite sport index. Brussels: Vrije Universiteit Brussel.

De Bosscher, V. et al. (2008b). A global sporting arms race: an nternational comparative study on sports policy factors leading to International sporting succés. Aachen: Meyer \& Meyer.

De Croock, S., De Bosscher, V. y van Bottenburg, M. (2012). The inspirational function of role models for sport participation and development. En: B. Soren y K. Morten, eds. Proceedings of the $20^{\text {th }}$ conference of the European Association of Sport. Aalborg: University College of Northern Denmark (UCN), 160.

EdComs, 2007. London 2012 legacy research: final report. London: COI/DCMS

Eichberg, H. et al., ed. (1998). Body cultures. Essays on sport, space and identity. London: Routledge.

Ewens, W. y Lashuk, M. (1989). Role model identification and activity preferences of ustralian youth. The ACPER national journal, 125, 18-20.

Feddersen, A., Jacobsen, S. y Maenning, W. (2009). Sports heroes and mass sports participation - the (double) paradox of the "German Tennis Boom". Available from: http://ssrn.com/abstract=1544320 (visto el 21 de febrero de 2018).

Fleming, S. et al. (2005). Role models' among elite young male rugby league players in Britain. European physical education review, 1 (1), 51-70. DOI: https://doi.org/10.1177/1356336X05049824

French, J. y Pena, S. (1991). Children's hero play of the 20th century: changes resulting from television's influence. Child study journal, 21 (2), 79-95.

Frawley, S. y Crush, A. (2011) Major sport events and participation legacy: the case of the 2003 Rugby World Cup. Managing leisure, 16, 65-76. DOI: https://doi.org/10.1080/13606719.2011.532605

Frick, B. y Wicker, P. (2016). The trickle-down effect: how elite sporting success affects amateur participation in German football. Applied Economics Letters, 23 (4), 259-263. DOI: https://doi.org/10.1080/13504851.2015.1068916

Girginov, V. y Hills, L. (2008). A sustainable sports legacy: creating a link between the London Olympics and sports participation. International journal of the history of sport, 25 (14), 2091-2116. DOI: https://doi.org/10.1080/09523360802439015

Girginov, V. y Hills, L. (2009). The political process of constructing a sustainable London Olympics sport development legacy. International journal of sport policy and politics, 1 (2), 161-181. DOI: https://doi.org/10.1080/19406940902950713

Globus, S. (1998). Atheltes as role models. Current health, 24 (6), 25-28. DOI: https://doi.org/10.1177/183335839802800114

Green, B.C. (2005). Building sport programs to optimize athlete recruitment, retention and transition: Towards a normative theory of sport development. Journal of sport management, 19, 233-253. DOI: https://doi.org/10.1123/jsm.19.3.233

Grix, J. y Carmichael, F. (2012). Why do governments invest in elite sport? A polemic. International journal of sport policy and politics, 4 (1), 73-90. DOI: https://doi.org/10.1080/19406940.2011.627358

Guiliano, T. et al. (2007). Gender and the selection of public athletic role models. Journal of sport behavior, 30 (20), 161-199

Hanstad, D. y Skille, E. (2010). Does elite sport devolop mass sport? Scandinavian sport studies forum, 1, 51-68 
Haut, J. y Gaum, C. (2017). Does elite success trigger mass participation in table tennis? An analysis of trickle-down effects in Germany, France and Austria. Journal of Sports Sciences, 35 (23), 1-8. DOI: https://doi.org/10.1080/02640414.2017.1361895

Heinilä, K. (1982). The totalisation process in International sport. Toward a theory of the totalization of competition in top-level sport. Sportwissenschaft, 3, 235-253.

Hindson et al., (1994). The trickle down effect of top level sport: myth or reality? A case study of the Olympics. Australian journal of leisure and recreation, 4 (1), $16-31$.

Houlihan, B. y Green, M. (2008). Comparative elite sport development. Systems, structures and public policy. London: Elsevier.

Jones, M.J. y Schumann, D.W. (2000). The strategic use of celebrity athlete endorsers in Sports lllustrated: an historic perspective. Sport marketing quarterly, 9 820), 65-76.

Jung, J. (1986). How useful is the concept of role model? A critical analysis. Journal of social behavior and personality, Journal of social behavior and personality, 1 (4), 525536.

Leopkey, B. y Parent, M. (2012). The (Neo) institutionalization of legacy and its sustainable governance within the Olympic Movement. European sport management quarterly, 12 (5), 437-456. DOI: https://doi.org/10.1080/16184742.2012.693116

Lines, G. (2011). Villains, fools or heroes? Sports stars as role models for young people. Leisure studies, 20 (4), 285-30. DOI: https://doi.org/10.1080/02614360110094661

Lockwood, P. y Kunda, Z. (1997). Superstars and me: predicting the impact of role models on the self. Journal of personality and social psychology, 73 (1), 91-103. DOI: https://doi.org/10.1037/0022-3514.73.1.91

London East Research Institute, University of East London, (2007). Lasting legay for London? Assessing the legacy of the Olympic games and Paralympic games. London: LERI.

Misener, L., Taks, M., Chalip, L. y Green, B.C. (2015). The elusive "trickle-down effect" of sport events: Assumptions and missed opportunities. Managing Sport and Leisure, 20 (2), 135-156.

Nauta, M. y Kokaly, M. (2001). Assessing role model influences on students' academic and vocational decisions. Journal of career assessment, 9 (1), 81-99. DOI: https://doi.org/10.1177/106907270100900106

PASW Statistics (Versión 17) [Programario informático]. Chicago, IL: SPSS Inc.

Payne, W. et al. (2003). Sports role models and their impact on participation in physical activity: a literature review, School of Human Movement and Sport Sciencies, University of Ballarat.

Renson, R. (2002). Inclusion or exclusion? Possibilities and limitations for interacton between sport for all and elit esport. En: NOC*NSF, ed. Proceedings van het 9de World Sport for All Congress. Sport for all and elit esport: Rivals or partners?. Ahrnem: NOC*NSF, 49.

Safrit, M. J., y Wood, T. M. (1995). Introduction to measurement in physical education and exercise science (3rd ed.). St. Louis, MO: Times Mirrow/Mosby.

Sotiriadou, P. y Shilbury, D. (2009). Australian elite athlte development: an organizational perspective. Sport management review, 12 (3), 137-148. DOI: https://doi.org/10.1016/j.smr.2009.01.002.

Sotiriadou, P., Shilbury, D., y Quick, S. (2008). The attraction, retention/transition and nurturing process of sport develpment: some Australian evidence. Journal of sport management, 22, 247-272. DOI: https://doi.org/10.1123/jsm.22.3.247

Steward, B. y Nicholson, M. (2004). The impact of elite sporting success on organized sport participation in Australia. En: E. de Boever, ed. Innovation through cooperation: proceedings of the European Sport Management congress, 22-25 September. Ghent: PVLO, 70-71.

Storm, R.K. y Asserhoj, T.L. (2016). The question of the trickle-down effect in Danish sport: Can a relationship between elite sport success and mass participation be 
identified?. En: Bailey, R. ed. Elite Sport and Sport-for-All. Bridging the Two Cultures?. Routledge, 41-56. DOI: https://doi.org/10.4324/9781315743059-4

Stokvis, R. y Minnee, R. (1986). De Olymplische Spelen van 1992 en de Georganiseerde Sport in Nederlan (The olypmic games of 1992 and the organised sport in the Netherlands). Spel en Sport, 3, 2-5.

van Bottenburg, M. (2001). Global games. Urbana: University of Illinois Press.

van Bottenburg, M. (2002). Sport for all and elite sport: do they benefit one another? En: NOC*NSF, ed. Proceedings of the 9th World Sport for All Congress, "Sport for all and elite sport: Rivals or partners?". Ahrnem: NOC*NSF, 25.

van Bottenburg, M. (2003). Top-en Breedtesport: een Siamese Tweeling? (Elite sport and sport for all: a Siamese twin). En: Breedveld, R. ed. Rapportage sport 2003. Den Haag: Sociaal en cultureel planbureau, 285-312.

van Bottenburg, M. et al. (2011). De Maatschappelijke Betekenis van Topsport (The social meaning of elite sport). Utrech: Mulier Instituut \& Universiteit Utrech.

Veal, A. et al. (2012). The sport participation legacy of the Sydney 2000 Olympic Games and other international sporting events hosted in Australia. Journal of policy research in tourism, leisure and events, 4 (2), 155-184. DOI: https://doi.org/10.1080/19407963.2012.662619

Veal, A. et al. (2012). The sport participation legacy of the Sydney 2000 Olympic Games and other international sporting events hosted in Australia. Journal of policy research in tourism, leisure and events, 4 (2), 155-184. DOI: https://doi.org/10.1080/19407963.2012.662619

Vescio, J. et al. (2005). Profiling sport role models to enhance initiatives for adolescent girls in physical education and sport. European physical education review, 11, 153170. DOI: https://doi.org/10.1177/1356336X05052894

Weimar, D., Wicker, P., y Prinz, J. (2015). Membership in nonprofit sport clubs: A dynamic panel analysis of external organizational factors. Nonprofit and voluntary sector quarterly, 44 (3), 417-436. DOI: https://doi.org/10.1177/0899764014548425

Wicker, P. et al. (2012). The value of Olympic success and the intengible effects of sport events -a contingent valuation approach in Germany. European sport management quarterly, 12 (4), 337-355. DOI: https://doi.org/10.1080/16184742.2012.693117

Número de citas totales / Total references: 55 (100\%)

Número de citas propias de la revista / Journal's own references: 0 (0\%)

Rev.int.med.cienc.act.fís.deporte - vol. 20 - número 77 - ISSN: 1577-0354 